\title{
Lohnhöhe, Güternachfrage und Beschäftigung
}

von Jürgen Jerger und Oliver Landmann

Juni 2000

\author{
Universität Freiburg \\ Platz der Alten Synagoge \\ D-79085 Freiburg \\ Tel.: ++49(0)761-203-2326 \\ Fax: ++49(0)761-203-2405 \\ email: jergerju | landmann@ vwl.uni-freiburg.de
}




\section{Lohnhöhe, Güternachfrage und Beschäftigung}

\section{Einleitung: konfuse beschäftigungspolitische Diskussion}

Ein Dauerbrenner der wirtschaftspolitischen Diskussion in der Bundesrepublik Deutschland ist die Debatte über die Frage, welchen Beitrag die Lohnpolitik bzw. die Geld- und Finanzpolitik zur Beseitigung der Arbeitslosigkeit leisten können und sollten. Diese Debatte lebt jedes Mal auf, wenn Tarifverhandlungen zwischen Arbeitgebern und Gewerkschaften anstehen, und sie verläuft immer nach demselben wiederkehrenden Ritual: Die Arbeitgeber argumentieren, dass die Anreize, neue Arbeitsplätze zu schaffen, entscheidend von den Arbeitskosten abhängen, und dass diese daher möglichst niedrig zu halten seien (Kostenargument). Die Gewerkschaften unterstreichen dagegen, dass sich die Nachfrage nach Arbeitskräften primär aus der effektiven Güternachfrage ableite, die ihrerseits durch eine steigende Kaufkraft der Löhne gestützt werde, im übrigen aber vor allem von der Geld- und Fiskalpolitik mit Rücksicht auf das Beschäftigungsziel zu steuern sei (Kaufkraftargument). Es ist verständlich, dass beide Seiten in den Lohnverhandlungen den Anschein zu erwecken suchen, nicht bloss ihr nacktes Eigeninteresse zu verfolgen, sondern auch die volkswirtschaftliche Vernunft auf ihrer Seite zu haben. Für frontal kollidierende Interessen müssen somit zwangsläufig ebenso frontal kollidierende Argumente in bezug auf die gesamtwirtschaftlichen Beschäftigungswirkungen der Lohnabschlüsse ins Feld geführt werden.

Wer hat Recht? Von der Wissenschaft wird erwartet, dass sie eine klare Antwort auf diese Frage anzubieten hat. Aber die Wissenschaft ist offenbar genauso zerstritten, wie es die Interessenvertreter in der Politik sind - oder dies ist zumindest das Bild, das sie derzeit nach aussen projiziert. Ein unvoreingenommener interessierter Laie, der sich mit Hilfe eines wirtschaftspolitischen Forums wie des Hamburger Wirtschaftsdiensts zu informieren sucht und dort z.B. einen Beitrag über das 'Ende der Bescheidenheit' (Berthold/Fehn/Thode in Heft 12/1998), einen Beitrag über die Beschäftigungswirkungen der Lohnzurückhaltung (Flassbeck in Heft 2/2000) sowie eine Debatte über einen möglichen Lohnsenkungswettbewerb in der Europäischen Währungsunion (Schürfeld, Kromphardt und Heise/Schulten in den Heften 9/1998 und 2/1999) gelesen hat, kann nur zum Schluss kommen, dass es offenbar für alle noch so widersprüchlichen Behauptungen über die Zusammenhänge zwischen Löhnen, Güternachfrage und Beschäftigung wissenschaftliche (oder wissenschaftlich klingende) Begründungen gibt. Nicht besser ergeht es ihm, wenn er das Jahresgutachten 1999/2000 des Sachverständigenrats zur Begutachtung der gesamtwirtschaftlichen Entwicklung in die Hand nimmt (Sachverständigenrat 1999) und dort von der Ratsmehrheit erfährt, dass am Arbeitsmarkt eine zurückhaltende Lohnpolitik viel, eine Belebung der Güternachfrage dagegen wenig bewirken könne, wogegen ein Minderheitsvotum des Ratsmitglieds Jürgen Kromphardt genau das Gegenteil behauptet. 
Einig ist man sich bestenfalls darüber, dass man mit dem Verhältnis zwischen Lohn- und Produktivitätswachstum argumentieren muss, wenn man über Beschäftigungswirkungen der Lohnpolitik spricht. Aber da ist der Konsens dann auch schon zu Ende. Das der Öffentlichkeit vermittelte Bild der heillosen Zerstrittenheit steht in einem merkwürdigen Kontrast zu der sehr weitgehenden Übereinstimmung, mit der die wechselseitigen Wirkungszusammenhänge zwischen Löhnen, Preisen, Kosten, Nachfrage und Beschäftigung in den Standard-Lehrbüchern der Makroökonomik dargestellt werden. Deshalb entwickeln wir im folgenden ein einfaches makroökonomisches Modell, das in seiner Struktur dem gängigen AS-AD-Paradigma der Lehrbücher sehr ähnlich ist, und stellen die Frage, welches Licht es auf die anhaltenden Kontroversen zu werfen vermag. Unter den Einsichten, die wir hierbei gewinnen, mag die eine oder andere auf den ersten Blick etwas überraschen. So zeigt sich insbesondere, dass weder die Relation von Lohnniveau und Arbeitsproduktivität noch der Kaufkrafteffekt von Lohnerhöhungen für die Beschäftigung jene kausale Bedeutung besitzen, die ihnen in der aktuellen Debatte gerne zugeschrieben wird.

In Abschnitt 2 entwickeln wir, anknüpfend an Landmann/Jerger (1999, Kap. 2), das Modell in einfachst möglicher Form und demonstrieren in Abschnitt 3 seinen Erklärungsgehalt für die Entwicklung der Beschäftigung in der Bundesrepublik Deutschland. In Abschnitt 4 erweitern wir das Modell um einen expliziten Kaufkrafteffekt der Löhne. Abschnitt 5 beschliesst den Beitrag mit einigen Schlussfolgerungen.

\section{Die Interaktion von Löhnen, Preisen, Güternachfrage und Beschäftigung}

Die Diskussion über die relative Bedeutung der Kosten- und Kaufkrafteffekte des Arbeitslohnes reicht, wie erwähnt, weit zurück. Ein Meilenstein war die General Theory von Keynes (1936), die erstmals überhaupt einen kohärenten analytischen Rahmen für diese Diskussion schuf. Später setzte man grosse Hoffnungen in die sog. Theorie der temporären Gleichgewichte, die in den 70er Jahren entwickelt wurde, und mit der sich zeigen liess, dass bei kurzfristig rigiden Preisen und Löhnen alternative Rationierungsregimes auftreten können, u.a. das Regime der keynesianischen Arbeitslosigkeit und das Regime der klassischen Arbeitslosigkeit (Malinvaud 1977). Im Falle des ersteren verursacht ein Mangel an effektiver Güternachfrage die Arbeitslosigkeit, im Falle des letzteren bildet ein überhöhtes Reallohnniveau den bindenden Engpass auf dem Arbeitsmarkt. Die Frage nach den Beschäftigungswirkungen einer Lohnänderung schien damit weitestgehend auf die Frage reduzierbar zu sein, in welchem Regime man sich gerade befindet (vgl. z.B. van Suntum 1997).

Eine wesentliche Schwäche dieses Fixpreis-Ansatzes liegt darin, dass er nur die Mengenreaktionen der Unternehmen auf exogene Änderungen (also z.B. Lohnänderungen) betrachtet, nicht aber die bei Kostenänderungen ebenfalls zu erwartenden Auswirkungen auf die von den Unternehmen geforderten Güterpreise. Stellt man die Endogenität der Güterpreise hingegen in Rechnung, so folgt unmittelbar, dass die Lohnpolitik, selbst wenn sie Niveau und Anstieg der Nominallöhne vollkommen bestimmen kann, keine direkte Kontrolle über die 
Reallöhne ausüben kann. Letztere sind ja als Quotient von Nominallohn- und Preisniveau definiert, und damit auch abhängig vom Preissetzungsverhalten der Unternehmen.

Einsicht 1: Die Lohnpolitik kann nur Nominallöhne festlegen. Die Reallöhne reflektieren demgegenüber auch die von den Unternehmen festgelegten Güterpreise. Es ist deshalb unzulässig, das Verhalten der Lohnpolitik an der Reallohnentwicklung zu messen, und sinnlos, der Lohnpolitik Vorgaben in Kategorien des Reallohns zu machen.

Am Rande sei vermerkt, dass auch die Nominallöhne durch die Tariflohnpolitik nur bedingt steuerbar sind, weil sich die effektiv gezahlten Löhne um die variable Lohnspanne von den Tariflöhnen unterscheiden. Um zu zeigen, welche weiteren Konsequenzen aus Einsicht 1 folgen, analysieren wir zunächst (entlang Solow 1986) die Entscheidungssituation, in der sich die Unternehmen befinden, wenn sie bei unvollkommenem Wettbewerb mit einer fallenden PreisAbsatz-Funktion konfrontiert sind und daher simultan über Absatzpreise, Güterproduktion und Arbeitseinsatz entscheiden müssen. Wir nehmen an, dass die Unternehmen ihren Output $Y$ mit Hilfe von Arbeit $N$ und Kapital $K$ produzieren. Um die Dinge etwas konkreter zu machen, unterstellen wir zudem die folgende Cobb-Douglas- Produktionstechnologie:

$$
Y=(A N)^{\alpha} \cdot K^{1-\alpha},
$$

wobei $A$ einen Indikator für den Stand der Technologie (bei arbeitsvermehrendem technischen Fortschritt) bezeichnet. Die Produktionselastizität der Arbeit ist durch $0<\alpha<1$ gegeben.

Für die Durchschnitts- und die Grenzproduktivität der Arbeit folgt

$$
\begin{aligned}
& Y / N=C N^{\alpha-1} \text { und } \\
& \partial Y / \partial N=\alpha C N^{\alpha-1} \quad \text { mit } C \equiv A^{\alpha} \cdot K^{1-\alpha} .
\end{aligned}
$$

Beide Produktivitätsmasse nehmen zu, a) wenn sich die Technologie verbessert, b) wenn der Kapitalbestand wächst, oder c) wenn die Beschäftigung zurückgeht. Die beiden erstgenannten Einflüsse sind in der Größe $C$ zusammengefasst. Gewinnmaximierende Unternehmungen wählen nun, wie aus der Preistheorie bekannt ist, die optimale Beschäftigungsmenge so, dass sich bei einer marginalen Variation der Beschäftigung Grenzerlös und Grenzkosten gerade die Waage halten (Amoroso-Robinson-Bedingung):

$$
P \cdot\left(1+\delta^{-1}\right)=\frac{W}{\partial Y / \partial N}
$$

Diese Bedingung bringt das Preissetzungsverhalten der Unternehmungen zum Ausdruck, denn sie impliziert unter Berücksichtigung von (3) den folgenden Zusammenhang zwischen Güterpreis $P$, Nominallohn $W$ und Beschäftigung $N$ :

$$
P=\frac{\mu}{\alpha} \cdot \frac{W}{C} \cdot N^{1-\alpha} \quad \text { mit } \mu \equiv \frac{1}{1+\delta^{-1}}>1 .
$$

Der Parameter $\delta$ (Annahme: $\delta<-1$ ) ist die Preiselastizität der Preis-Absatz-Funktion des repräsentativen Unternehmens; er könnte aber auch andere Abweichungen vom Paradigma des vollkommenen Wettbewerbs abbilden, die einen Keil zwischen den Reallohn und die Grenzproduktivität der Arbeit treiben (Blanchard 1998, S. 6). Das Preisniveau $P$ kann somit als 
Aufschlag $\mu$ auf die Grenzkosten $\frac{W}{\partial Y / \partial N}=\frac{W}{\alpha C} \cdot N^{1-\alpha}$ bzw. als Aufschlag $\mu / \alpha$ auf die Lohnstückkosten $\frac{W N}{Y}=\frac{W}{C} \cdot N^{1-\alpha}$ aufgefasst werden. Schon an dieser Stelle können wir feststellen, dass mit dem Preissetzungsverhalten (4) - unabhängig davon, wie die Lohnpolitik den Nominallohn $W$ steuert - die gesamtwirtschaftliche Lohnquote $\Lambda \equiv \frac{W N}{P Y}$ bestimmt ist:

$$
\Lambda \equiv \frac{W N}{P Y}=\frac{W}{P} \cdot C^{-1} \cdot N^{1-\alpha}=\frac{\alpha}{\mu} .
$$

Einsicht 2: Die Lohnquote ist kein Ergebnis der Lohnpolitik, sondern reflektiert die Produktionselastizität der Arbeit $(\alpha)$ sowie diejenigen Marktunvollkommenheiten, die einen Keil zwischen den Reallohn und die Grenzproduktivität der Arbeit treiben $(\mu)$. Dies bedeutet insbesondere, dass z.B. ein Rückgang der Lohnquote oder, was dasselbe ist, ein Zurückbleiben des Reallohnanstiegs hinter dem Anstieg der Arbeitsproduktivität nicht als Indiz für eine zurückhaltende Lohnpolitik interpretiert werden können.

$\mathrm{Zu}$ relativieren ist diese Einsicht allenfalls in zweierlei Hinsicht: Zum einen bedeutet jede Verzögerungen, mit der sich die Güterpreise und/oder die Beschäftigung nach einer Nominallohnänderung an die von Bedingung (4) diktierten Werte anpassen, immer auch eine vorübergehenden Abweichung der Lohnquote von ihrem Gleichgewichtswert $\alpha / \mu$. Zum anderen kann die Lohnpolitik einen Einfluss auf die Produktionselastizität $\alpha$ ausüben, wenn die Produktionstechnologie nicht den Restriktionen der von uns einfachheitshalber gewählten CobbDouglas-Spezifikation gehorcht. Dieser Einfluss hängt von der Substitutionselastizität zwischen Arbeit und den anderen Produktionsfaktoren sowie von der induzierten Reaktion der Kapitalakkumulation ab und kann in die eine oder die andere Richtung gehen.

Um das Modell zu schliessen, müssen wir spezifizieren, wie sich auf der gesamtwirtschaftlichen Ebene die Güternachfrage bildet. Der einfachste Weg, dies zu tun, besteht darin, die Quantitätstheorie des Geldes heranzuziehen und die nominale Gesamtnachfrage $P Y$ als das Produkt von Geldmenge $M$ und Umlaufgeschwindigkeit $V$ zu schreiben:

$$
P Y=M V \quad \text { bzw. } Y=M V / P
$$

Wir behandeln das Produkt $M V$ zunächst einmal als exogene Grösse, weisen aber bereits hier darauf hin, dass diese Darstellung der Nachfrageseite durch geeignete funktionale Spezifikation der Umlaufgeschwindigkeit $V$ beliebig verfeinert - z.B. auch in Einklang mit einer keynesianischen IS-LM-Modellierung der Nachfragebestimmung gebracht - werden kann. Auf diesen Punkt werden wir im nächsten Abschnitt im Zusammenhang mit der Kaufkrafttheorie der Löhne zurückkommen.

Die Gleichungen (1), (4) und (6) bestimmen nun zusammen Output, Beschäftigung und Preisniveau. Die zugrundeliegende Logik ist sehr einfach: Die Unternehmen setzen Güterpreise nach Massgabe von Gleichung (4), bedienen die Nachfrage, die sich gemäss (6) bei diesem Preisniveau einstellt, und beschäftigen die Arbeitskräfte, die gemäss der Produktionsfunktion (1) für die entsprechende Güterproduktion erforderlich sind. Für die weitere Diskussion des Modells ist es hilfreich, diese drei Gleichungen durch Logarithmieren in ein (log-) lineares System 
überzuführen, wobei kleine Buchstaben jeweils den natürlichen Logarithmus bezeichnen. Aus (1) ergibt sich unmittelbar die Produktionsfunktion (PF)

(1') $y=\alpha \cdot(a+n)+(1-\alpha) \cdot k \equiv c+\alpha n \quad$ mit $c=\log C$.

Aus (4) ergibt sich nach Elimination von $n$ mit Hilfe von (1') die Preissetzungsfunktion (PS)

(4') $\quad p=w-c-\lambda+\frac{1-\alpha}{\alpha} \cdot(y-c)$ mit $\lambda \equiv \log \Lambda$

Viele Lehrbücher nehmen vollkommene Konkurrenz auf den Gütermärkten an $(\mu=1)$, lösen aufgrund des von dieser Annahme implizierten Mengenanpasserverhaltens der Unternehmungen Beziehung (4') nach dem Output $y$ auf und bezeichnen das Ergebnis als 'Aggregierte Angebotsfunktion'. Aus Gleichung (6) resultiert unmittelbar die Aggregierte Nachfragefunktion $(\mathrm{AN})$ :

(6') $y=m+v-p$

Abbildung 1 veranschaulicht grafisch, wie die drei Beziehungen (1'), (4') und (6') gemeinsam Output, Beschäftigung und Preisniveau bestimmen. Die algebraische Lösung lautet wie folgt:

$$
\left[\begin{array}{l}
y \\
n \\
p
\end{array}\right]=\left[\begin{array}{cccc}
\alpha & -\alpha & \alpha & 1 \\
1 & -1 & 1 & 0 \\
1-\alpha & \alpha & -\alpha & -1
\end{array}\right]\left[\begin{array}{c}
m+v \\
w \\
\lambda \\
c
\end{array}\right]
$$




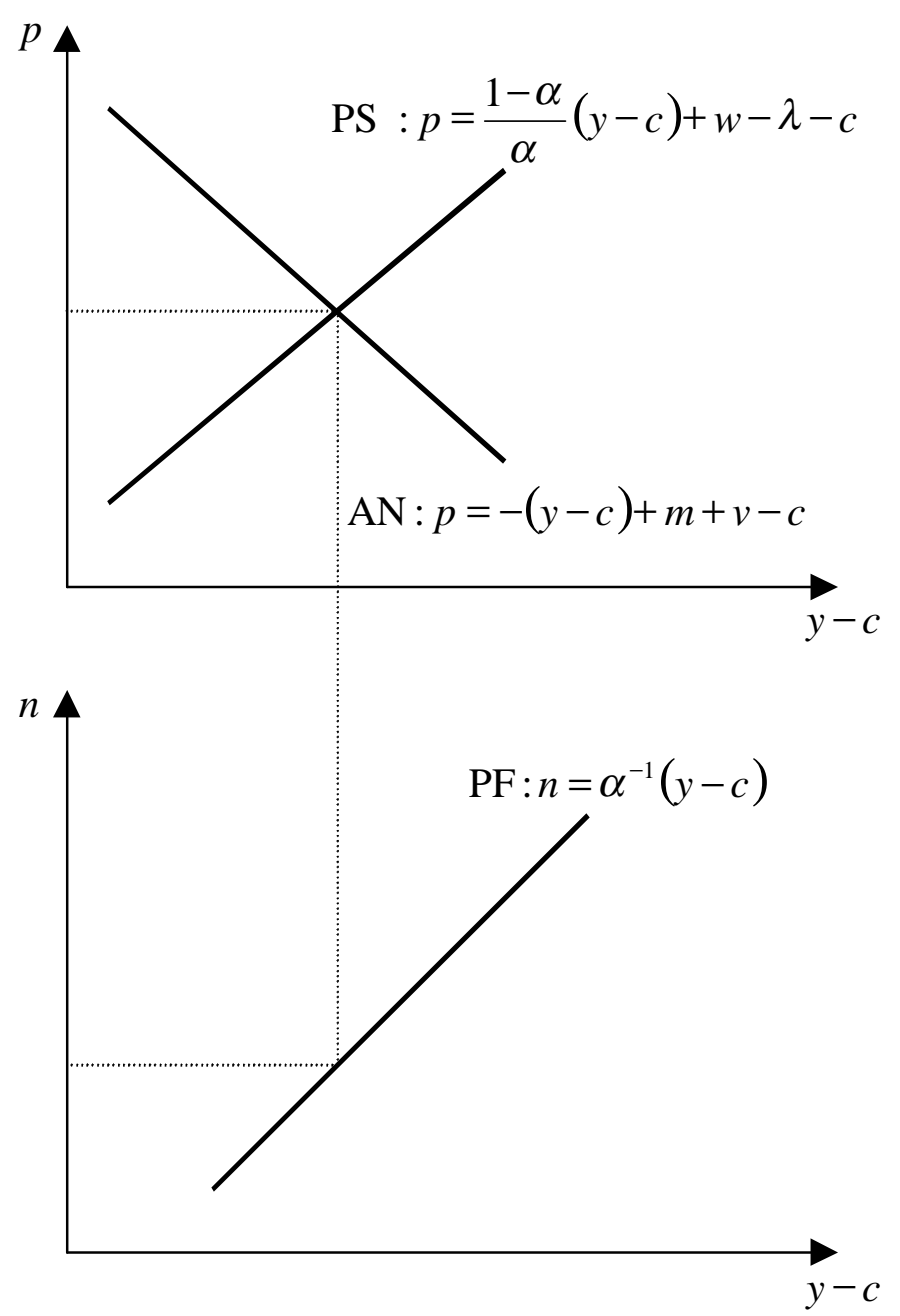

Abbildung 1

Die Gleichgewichtslösung (7) kann als Wachstumsgleichgewicht bei konstanter Beschäftigung im Sinne des neoklassischen Wachstumsmodells (Solow 1956) gedeutet werden. Ein solches ist dadurch charakterisiert, dass der Kapitalstock $K$ mit der Rate des arbeitsvermehrenden technischen Fortschritts wächst ( $d k / d t=d a / d t \equiv q)$, womit gemäss Definition von $c$ auch gilt: $d c / d t=q$. In einem Wachstumsgleichgewicht mit konstanter Beschäftigung wachsen daher auch die Arbeitsproduktivität und der Output mit der technischen Fortschrittsrate $q$. Die gleichgewichtige Wachstumsrate $q$ wird oft auch als Trendproduktivitätswachstum oder beschäftigungsneutraler Fortschritt der Arbeitsproduktivität bezeichnet. Wie aus (1') hervorgeht, bedeutet ein Beschäftigungsrückgang, dass die Arbeitsproduktivität vorübergehend rascher wächst als mit der Rate $q$ - ein Produktivitätsbonus, der vom Sachverständigenrat (1999, Ziff. 334) als "Entlassungsproduktivität" bezeichnet wird, weil er darauf beruht, dass es immer die am wenigsten produktiven Arbeitsplätze sind, die einem Beschäftigungsabbau als erste zum Opfer fallen.

In Abbildung 1 ist auf den beiden Abszissen der um den Produktivitätstrend bereinigte Output $(y-c)$ abgetragen. Damit lässt sich eine Situation, in der die Volkswirtschaft mit der Rate $q$ 
wächst, als stationäres Gleichgewicht darstellen. Der untere Quadrant illustriert die in unserem Zusammenhang zentrale Implikation der Produktionsfunktion (1`): Die Beschäftigung $n$ kann bei jedem gegebenen Wert von $\alpha$ nur dann zunehmen, wenn der bereinigte Output $(y-c)$ steigt. Dieser wiederum kann, wie der obere Quadrant deutlich macht, nur steigen, wenn sich die Preissetzungsfunktion (PS) und die Aggregierte Nachfragefunktion (AN) relativ zueinander so bewegen, dass sich ihr Schnittpunkt nach rechts verlagert. Die Bedingung, unter der dies geschieht, lässt sich aus Abbildung 1 ebenso ablesen wie aus der algebraischen Modellösung (7): Die nominale Gesamtnachfrage $m+v$ muss (für jeden gegebenen Wert von $\lambda$ ) mit einer höheren Rate wachsen als das Nominallohnniveau $w$. Der Trendproduktivitäts-Term $c$ geht demgegenüber nicht in die Bestimmungsgleichung der Beschäftigung ein. Dies bedeutet auch, dass die Veränderung des Nominallohns relativ zum Produktivitätswachstum für das Verhalten der Beschäftigung überhaupt nichts präjudiziert. Die so oft beschworene Produktivitätsregel, wonach die Nominallohnzuwächse im Ausmass des Produktivitätsfortschritts zu halten seien, ist weder eine notwendige noch eine hinreichende Bedingung für die Stabilisierung der Beschäftigung.

Einsicht 3: Die Beschäftigungsmenge ist durch die Relation zwischen nominaler Güternachfrage und Nominallohn bestimmt. Das Produktivitätswachstum hat demgegenüber keinen direkten Einflu $\beta$ auf die Beschäftigung. Eine Lohnpolitik, die den Anstieg der Nominallöhne im Rahmen des Trendproduktivitätswachstums hält, ist weder eine notwendige noch eine hinreichende Bedingung für die Stabilisierung der Beschäftigung.

Die Produktivitätsregel $d w / d t=q$ gewährleistet lediglich, dass die PS-Funktion im oberen Quadranten von Abbildung 1 stationär bleibt - und selbst dies gilt nur, wenn sich $\lambda$ nicht verändert. Damit die Beschäftigung bei Einhaltung der Produktivitätsregel konstant bleibt, muss zudem auch die AN-Funktion stabil bleiben, was genau dann der Fall ist, wenn die nominale Gesamtnachfrage mit der Rate des Trendproduktivitätswachstums $q$ wächst $(d m / d t+d v / d t=q)$. Die Verbindung einer solchen potentialorientierten Nachfragesteuerung mit einer Lohnpolitik, die sich an die Produktivitätsregel hält, ist mithin das Rezept für die simultane Stabilität von Preisniveau und Beschäftigung. Aber nochmals: Die Produktivitätsregel allein gewährleistet weder das eine noch das andere, sondern verhindert bloss, dass von der Lohnpolitik ein positiver oder negativer - Impuls auf die Preissetzung ausgeht.

Etwas komplizierter stellt sich die Situation dar, wenn die Lohnquote $\lambda$ entgegen unserer bisherigen Annahme nicht konstant ist, sondern selbst einem Trend unterliegt. Die Absenz eines solchen Trends zählt zwar - über sehr lange Zeiträume hinweg gesehen - zu den sog. 'stilisierten Fakten' des Wachstumsprozesses. Dennoch ist es eine Tatsache, dass die Lohnquote in der Bundesrepublik Deutschland wie auch in anderen europäischen Volkswirtschaften nach einem starken Anstieg in den 70er Jahren während der letzten ca. 20 Jahre mehr oder weniger stetig zurückgegangen ist. Die Reallohnsteigerungen dieser Periode haben mit anderen Worten nicht ausgereicht, die Arbeitnehmer in vollem Umfang am Produktivitätsfortschritt zu beteiligen. Dass hierin nicht, wie häufig behauptet, ein Indiz einer zurückhaltenden Lohnpolitik gesehen werden kann, sondern dass längerfristige Veränderungen von $\lambda$ auf Veränderungen der Produktionselastizität der Arbeit, der Marktmacht der Unternehmer oder anderer Marktverzerrungen zurückzuführen sind, haben wir bereits oben festgestellt. Was den Rückgang während der letzten beiden Jahrzehnte im einzelnen verursacht hat, ist nicht restlos klar (Blanchard 1997, 1998). Klar ist gemäss Gleichung (7) hingegen, dass jeder Rückgang von $\lambda$ auf die Preissetzungsfunktion genau gleich wirkt wie ein Anstieg des Nominallohns und insofern den Spielraum für Lohnerhöhungen, die ohne negative Rückwirkungen auf die Beschäftigung 
gewährt werden können, verringert (vgl. Lehment 1999, Sachverständigenrat JG 1998/99, Anhang IV.E). Unter Berücksichtigung von Veränderungen der Lohnquote lautet die Bedingung für die Konstanz der Beschäftigung $d w / d t=d(v+m) / d t+d \lambda d t$.

\section{Ein Blick auf die Daten}

Die zentrale Botschaft unseres Modells lautet, dass die Beschäftigung proportional mit dem Quotienten von nominaler Gesamtnachfrage und Nominallohn variiert. Was hieraus für die Änderungsraten dieser Grössen folgt, wird deutlich, wenn wir die Beschäftigungsgleichung der Modellösung (7) nach der Zeit ableiten:

(7`) $\frac{d n}{d t}=\frac{d(m+v)}{d t}-\frac{d w}{d t}+\frac{d \lambda}{d t}$

Wie aber haben sich diese Wachstumsraten in der Bundesrepublik Deutschland tatsächlich zueinander verhalten? Abbildung 2 zeigt das jährliche Beschäftigungswachstum als durchgezogene Linie und die Differenz der nominalen Wachstumsraten der Gesamtnachfrage und des Lohnniveaus als gestrichelte Linie.

\section{Abbildung 2 etwa hier}

Es ist gut zu sehen, dass der von der Theorie prognostiziere Zusammenhang von den Daten klar bestätigt wird. Die Beobachtung, dass die beiden Zeitreihen vertikal etwas gegeneinander versetzt sind, reflektiert den oben angesprochenen Zeittrend der Lohnquote $\lambda$. Natürlich ist die Korrelation von Jahr zu Jahr keineswegs perfekt. Mögliche Gründe für diese Abweichungen sind (unter anderem)

- Verzögerungen in der Anpassung der Beschäftigung an den Optimalwert, den das vom Modell unterstellte unternehmerische Optimierungskalkül impliziert. Dafür verantwortlich können beispielsweise Kündigungsschutzregelungen oder andere Kosten der Beschäftigungsanpassung sein, die zu einer Glättung der Beschäftigungsschwankungen relativ zu den konjunkturellen Schwankungen der Güternachfrage führen.

- Unvollkommene Anpassung der Beschäftigung an die Arbeitsnachfrage bei MismatchArbeitslosigkeit (Friktionen bei der Zusammenführung von offenen Stellen und Arbeitsuchenden).

- Verzögerungen in der Anpassung der Güterpreise an das Niveau, das gemäß Gleichung (4') von den Lohnkosten und der Güternachfrage her angezeigt wäre.

\section{[Überlegen: Regressionsgleichung bringen, um nachzuweisen, dass sich über die Lags hinweg die Proportionalität bestätigt?]}

Trotz dieser Einwände macht Abbildung 2 deutlich, dass die simple Version eines makroökonomischen Lehrbuch-Modells der aggregierten Nachfrage und der aggregierten Preissetzung, die wir im vorigen Abschnitt entwickelt haben, die Realität keineswegs in unzulässiger oder irreführender Weise übervereinfacht. Dabei ist es auch wichtig, sich klar zu machen, dass die enge Korrelation der beiden Zeitreihen in Abbildung 2 nicht etwa daher rührt, dass der behauptete Zusammenhang rein tautologischer Natur wäre. Dieser Verdacht könnte 
vielleicht aufkommen, wenn man sich vergegenwärtigt, dass Gleichung (7') so, wie sie oben geschrieben steht, allein aus den Definitionsgleichungen der Umlaufgeschwindigkeit $v$ und der Lohnquote $\lambda$ ableitbar ist und insofern immer genau erfüllt sein muss. Aber das Modell behauptet ja wesentlich mehr. Sein Informationsgehalt liegt in der Vorhersage, dass sich Änderungen der Geldmenge, der Umlaufgeschwindigkeit und des Nominallohns in entsprechenden Änderungen der Beschäftigung niederschlagen - und nicht etwa in Änderungen der Lohnquote, wie dies rein logisch aufgrund der Definitionszusammenhänge ebenfalls möglich wäre. Und genau diese falsifizierbare Vorhersage ist es, die wir in Abbildung 2 in so hohem Masse verifiziert finden.

Die Bedeutung des Quotienten von nominaler Güternachfrage und Nominallohn für das Verhalten der Beschäftigung wurde früher schon in mehreren Papieren von Lehment nachgewiesen (zuletzt etwa Lehment 1999). Unser Erklärungsmodell zeigt die makrotheoretischen Grundlagen des Zusammenhangs explizit auf und verdeutlicht damit insbesondere auch, dass es irreführend ist, den erklärenden Quotienten als ein Mass der "Lohnzurückhaltung" zu interpretieren, wie dies gelegentlich geschieht (z.B. Lehment 1991, Lapp/Lehment 1997). Vielmehr gehen die Nachfrage- und die Lohnvariable völlig symmetrisch in den Quotienten ein, was gerade die gemeinsame Verantwortung der Lohnpolitik und der Nachfragepolitik für die Beschäftigung unterstreicht.

\section{Der Kaufkrafteffekt der Löhne}

Was unser Modell bisher vernachlässigt hat, ist das Argument, dass Löhne in einer Volkswirtschaft nicht nur ein Kostenfaktor sind, sondern auch ein Nachfragefaktor. Es stellt sich damit die Frage, ob und was sich an unseren bisherigen Ergebnissen ändert, wenn wir explizit berücksichtigen, dass Löhne zumindest teilweise nachfragewirksam ausgegeben werden und infolgedessen im Zähler des Nachfrage-Nominallohn-Quotienten ebenso wirksam werden wie im Nenner. Um die Konsequenzen dieses Effekts analysieren zu können, verallgemeinern wir die aggregierte Nachfragefunktion (6') wie folgt:

$$
y=\beta_{0} d+\beta_{1}(m-p)+\beta_{2}(w+n-p), \quad \text { mit } \beta_{0}, \beta_{1}, \beta_{2}>0 \text { und } \beta_{2}<\alpha,
$$

Die wiederum log-lineare Nachfragefunktion (6") lässt sich am besten als reduzierte Form eines IS-LM-Modells auffassen, in dessen IS-Gleichung die Variable $d$ die autonomen Nachfragekomponenten (Staatsausgaben, autonome Investition etc.) zusammenfasst, zusätzlich aber auch die (logarithmierte) reale Lohnsumme $(w+n-p)$ als eigene Nachfragedeterminante in Erscheinung tritt. Die Restriktion $\beta_{2}<\alpha$ stellt sicher, dass der in (6") angelegte keynesianische Einkommensmultiplikator einen endlichen Wert aufweist bzw. derMultiplikatorprozess konvergiert. Die hier gewählte Spezifikation ist zwar "ad hoc", d.h. nicht aus einer sorgfältigen Mikrofundierung des Verhaltens der Akteure abgeleitet. In Jerger/Michaelis (2000) wird jedoch gezeigt, dass die Annahme unterschiedlicher gruppenspezifischer Sparquoten von Arbeitnehmern und Unternehmern in einer zu (6") analogen aggregierten Nachfragefunktion resultiert.

Da sich durch die Berücksichtigung des Kaukrafteffekts der Löhne weder an dem Preissetzungskalkül der Unternehmer noch an der Produktionsfunktion etwas ändert, können die Gleichungen (1') und (4') zusammen mit (6") zur Berechnung der Lösung von $y, n$ und $p$ herangezogen werden. Diese lautet: 


$$
\left[\begin{array}{c}
y \\
n \\
p
\end{array}\right]=\Delta^{-1} \cdot\left[\begin{array}{ccccc}
\alpha \beta_{1} & -\alpha \beta_{1} & \alpha\left(\beta_{1}+\beta_{2}\right) & \beta_{1} & \alpha \beta_{0} \\
\beta_{1} & -\beta_{1} & \beta_{1}+\beta_{2} & \beta_{1}+\beta_{2}-1 & \beta_{0} \\
\beta_{1}(1-\alpha) & \alpha\left(1-\beta_{2}\right) & \beta_{2}-\alpha & \beta_{2}-1 & \beta_{0}(1-\alpha)
\end{array}\right] \cdot\left[\begin{array}{c}
m \\
w \\
\lambda \\
c \\
g
\end{array}\right]
$$

mit $\Delta \equiv \alpha\left(1-\beta_{1}-\beta_{2}\right)+\beta_{1}>0$

Diese Lösung vermittelt uns hinsichtlich der Beschäftigungswirkung einer Nominallohnänderung zwei Einsichten:

Einsicht 4a: Eine Nominallohnerhöhung führt auch unter voller Berücksichtigung ihres Kaufkrafteffekts immer zu einer Verminderung der Beschäftigung (ceteris paribus).

Einsicht 4b: Der negative Beschäftigungseffekt einer Nominallohnerhöhung ist umso grösser, je stärker der Kaufkrafteffekt ins Gewicht fällt.

Einsicht 4a folgt unmittelbar aus der Beschäftigungsgleichung in (9): $\partial n / \partial w=-\beta_{1} \Delta^{-1}<0$, was bedeutet, dass der Kosteneffekt den Kaufkrafteffekt einer Lohnänderung dominieren muss. Warum dies so ist, lässt sich am anschaulichsten unter Rückgriff auf Abbildung 1 begründen. Der Kaufkrafteffekt bedeutet ja einfach, dass eine Zunahme des Nominallohns nicht nur die Preissetzungsfunktion nach oben verschiebt, sondern auch die aggregierte Nachfragefunktion. Während sich die Preissetzungsfunktion aber gemäss Gleichung (4') genau im Ausmass der Lohnsteigerung nach oben verschiebt, verlagert sich die aggregierte Nachfragefunktion, wie die Inspektion der verallgemeinerten Nachfragefunktion (6") offenbart, in jedem Fall weniger weit nach oben. Der Schnittpunkt der Preissetzungsfunktion mit der aggregierten Nachfragefunktion verschiebt sich somit nach links oben, die Beschäftigung sinkt.

Einsicht $4 \mathrm{~b}$ besagt, dass die Ableitung der Beschäftigungsgleichung in (9) nach dem Nominallohn, $\partial n / \partial w$, dem Betrage nach umso grösser (d.h. algebraisch umso kleiner) wird, je grösser der Parameter $\beta_{2}$ ist. Dies folgt formal aus der Tatsache, dass der Parameter $\beta_{2}$ mit einem negativen Vorzeichen in die Determinante $\Delta$ eingeht. Die Intuition hinter diesem Ergebnis ist recht einfach: Eine Lohnerhöhung bedeutet zwar, für sich genommen, mehr Kaufkraft für die Beschäftigten. Andererseits wirken aber der Anstieg des Preisniveaus und der Rückgang der Beschäftigung, die als Folge der Lohnerhöhung eintreten, auf eine Schmälerung der realen Lohnsumme hin. Eine genauere Inspektion der Gesamtwirkung offenbart

$$
\frac{\partial(w+n-p)}{\partial w}=1+\frac{\partial n}{\partial w}-\frac{\partial p}{\partial w}=-\alpha \beta_{1} \Delta^{-1}<0 .
$$

Die Ungleichung (10) könnte man als das Kaufkraftparadoxon des Lohnes bezeichnen: Eine nominale Lohnerhöhung senkt die reale Kaufkraft der Lohnempfänger! Einsicht $4 \mathrm{~b}$ ist eine unmittelbare Implikation dieses Kaufkraftparadoxons.

Die Vorstellung, dass Lohnerhöhungen durch Stützung der Binnenkaufkraft einen Beitrag zur Verbesserung der Beschäftigungslage leisten könnten, erweist sich somit als falsch. Da das negative Vorzeichen von $\partial n / \partial w$ entscheidend durch die induzierte Reaktion des

Güterpreisniveaus bedingt ist, bleibt immerhin noch die Möglichkeit offen, dass über einen kürzeren Zeithorizont, innerhalb dessen die Anpassung der Güterpreise noch nicht abgeschlossen ist, ein vorübergehender positiver Beschäftigungseffekt resultiert. In Jerger (1999, Kapitel 2) 
wird diese theoretische Möglichkeit aufgezeigt, ein empirischer Test mit Quartalsdaten führt aber zu dem Ergebnis, dass die Anpassungsprozesse offenbar so schnell ablaufen, dass auch kurzfristig keine positiven Beschäftigungseffekte auftreten.

Im Ergebnis können wir festhalten, dass die Berücksichtigung eines Kaufkrafteffekts der Löhne an Einsicht 3 nichts ändert: Entscheidend für die Beschäftigung bleibt das Verhältnis zwischen der nominalen Gesamtnachfrage und dem Nominallohnniveau. Im Unterschied zu der kruden 'quantitätstheoretischen' Nachfragefunktion (6') macht die allgemeinere Spezifikation (6") bloss deutlich, dass eine Nachfragepolitik, die sich der Instrumente der Geld- und Fiskalpolitik bedient, auf die Kenntnis der Parameter $\beta_{0}$ und $\beta_{1}$ angewiesen ist und möglicherweise auch einem Einfluss der Lohnpolitik auf die nominale Gesamtnachfrage Rechnung tragen muss. In welche Richtung ein solcher Einfluss geht, ist allerdings nicht a priori klar. Gemäss der Modellösung (9) ist die Reaktion der nominalen Gesamtnachfrage auf Änderungen des Nominallohns gegeben durch

$$
\frac{\partial(p+y)}{\partial w}=\alpha\left(1-\beta_{1}-\beta_{2}\right) \Delta^{-1}
$$

was je nach Vorzeichen des Klammerausdrucks positiv oder negativ sein kann. Im Grenzfall $\beta_{1}+\beta_{2}=1$ ist die nominale Gesamtnachfrage unabhängig von der Entwicklung der Nominallöhne. Der ökonometrische Befund von Lehment (1999), wonach die Lohnentwicklung höchstens eine vorübergehende Wirkung auf das nominale Nachfragewachstum ausübt, spricht dafür, dass dieser Grenzfall nicht allzu weit von der Wirklichkeit entfernt sein dürfte -

\section{BESSER WÄRE ALLERDINGS, WENN WIR UNS HIER AUF JERGER (1999) BERUFEN KÖNNTEN!}

\section{Zusammenfassung und Schlußfolgerungen}

Sowohl bei den Tarifparteien als auch in der öffentlichen wirtschaftspolitischen Debatte herrscht bisweilen eine beträchtliche Konfusion um die Wirkungen der Lohnpolitik und um die adäquate Rollenverteilung zwischen Nachfragepolitik und Lohnpolitik. Dabei geht es vor allem um zwei Fragen: Erstens, an welcher Richtgröße sollten sich die Nominallohnabschlüsse orientieren, wenn die Tarifparteien einen Beitrag zur Erhöhung der Beschäftigung leisten wollen? Zweitens, welche Bedeutung sollte hierbei den Wirkungen der Lohnentwicklung auf die Binnennachfrage beigemessen werden?

Hinsichtlich der ersten Frage scheint es einen breiten Konsens darüber zu geben, dass eine Orientierung der Lohnpolitik am langfristigen Wachstum der Arbeitsproduktivität - d.h. an dem Produktivitätswachstum, das sich unter Herausrechnung der 'Entlassungsproduktivität' ergibt beschäftigungsneutral ist. Nicht immer klar ist, ob damit die Reallöhne oder die Nominallöhne gemeint sind. Der Lohnpolitik Vorgaben für die Reallöhne zu machen, ist unsinnig, weil die Lohnpolitik die Reallohnentwicklung nicht kontrolliert. Ein Zurückbleiben der Nominallöhne gegenüber dem Trendproduktivitätswachstum ist, so viel zeigt unser makroökonomisches Nachfrage-Preissetzungs-Modell, für eine Zunahme der Beschäftigung weder notwendig noch hinreichend. Worauf es ankommt, ist vielmehr die Relation zwischen dem Nominallohnanstieg und dem Wachstum der nominalen Gesamtnachfrage. Immerhin eignet sich das Produktivitätswachstum als gemeinsame Richtschnur für die Lohnpolitik und die 
Nachfragepolitik im Hinblick auf die simultane Stabilisierung des Preisniveaus und der Beschäftigung.

Die zweite Frage ist mit dem gängigen Lehrbuch-Modell nicht direkt analysierbar, weil eine eigenständige Rolle der Löhne bei der Bestimmung der aggregierten Nachfrage in diesem Modell nicht angelegt ist. Auch in der Forschung hat diese Frage bemerkenswert wenig Beachtung gefunden. Dabei lässt sich das Grundmodell relativ einfach um einen expliziten Einfluss der Lohnkaufkraft auf die gesamtwirtschaftliche Nachfrage erweitern. Wie die Analyse des erweiterten Modells allerdings zeigt, ändert der Kaufkrafteffekt der Löhne nichts am Ergebnis, dass das Wachstum der Nominallöhne unter dem Wachstum der nominalen Gesamtnachfrage liegen muss, wenn die aggregierte Beschäftigungsmenge steigen soll.

\section{Literatur}

Blanchard, Olivier (1997): "The Medium Run", Brookings Papers on Economic Activity 2:1997, S. 89141.

Blanchard, Olivier (1998): "Revisiting European Unemployment: Unemployment, Capital Accumulation and Factor Prices", NBER Working Paper 6566, Mai.

Jerger, Jürgen (1999): Nachfragesteuerung, Lohnbildung und Beschäftigung. Tübingen.

Jerger, Jürgen/Michaelis, Jochen (2000): "Nominal Wages, Real Demand and Aggregate Employment", Beiträge zur wirtschaftswissenschaftlichen Forschung Nr. 10, Februar 2000, Universität ErlangenNürnberg.

Landmann, Oliver/Jerger, Jürgen (1999): Beschäftigungstheorie. Berlin und Heidelberg.

Lapp, Susanne/ Lehment, Harmen (1997): "Lohnzurückhaltung und Beschäftigung in Deutschland und in den Vereinigten Staaten", Die Weltwirtschaft (1), S. 67-83.

Lehment, Harmen (1991): "Lohnzurückhaltung, Arbeitszeitverkürzung und Beschäftigung. Eine empirische Untersuchung für die Bundesrepubilk Deutschland 1973-1990." Die Weltwirtschaft (2), S. $72-85$

Lehment, Harmen (1999): "Zur Bestimmung des beschäftigungsneutralen Lohnerhöhungsspielraums", Die Weltwirtschaft (1), S. 79-89.

Malinvaud, Edmond (1977): The Theory of Unemployment Reconsidered, Oxford.

Sachverständigenrat (1999): Wirtschaftspolitik unter Reformdruck. Jahresgutachten 1999/2000. Stuttgart. Sachverständigenrat (1998): Vor weitreichenden Entscheidungen. Jahresgutachten 1998/1999. Stuttgart. Solow, Robert M. (1956): "A Contribution to the Theory of Economic Growth", Quarterly Journal of Economics, S. 65-94.

Solow, Robert M. (1986): "Unemployment: Getting the Questions Right”, Vol. 53, Supplement, S. S23S34.

van Suntum, Ulrich (1997): "Kaufkrafttheorie des Lohnes", WISU, Heft 1, S. 71-78. 